\title{
THE HOSPITALLERS' ACCOUNTS FOR 1373/4 AND 1374/5: AN ARAGONESE TEXT
}

\author{
Anthony Luttrell
}

A considerable number of Aragonese travelled or established themselves abroad during the fourteenth century, yet their language was little used, or at least seldom written, in Sicily, Greece or other places in which there was an Aragonese presence but where Catalans and the Catalan tongue were more dominant. The most notable exception was the series of translations and compilations produced at Avignon between about 1370 and 1396 for the Aragonese Hospitaller Fr. Juan Fernández de Heredia, who in 1377 became Master of the Hospital of Rhodes. His personal letters and the documents in the registers of his Hospitaller priories were often in Aragonese and there were evidently one or more scribes in his household at Avignon who wrote in that language. In 1374 Fernández de Heredia was the papal Captain-General of Avignon and the unofficial representative there of the Aragonese king, while he was also the Hospitallers' Prior in both Aragon and Catalunya and the Lieutenant in the West of the Master of Rhodes'.

The Hospital made use of Fernández de Heredia's financial

1 A. Luttrell, «Juan Fernández de Heredia and the Compilation of the Chronicle of the Moreas, in The Aragonese Version of the Chronicle of the Morea, ed. A. Luttrell - D. Mackenzie, Madison 1985. 
capabilities. In 1373 an assembly of Hospitallers at Avignon agreed, somewhat unrealistically, to raise large sums by imposing extraordinary taxes in order to finance a passagium against the Turks. There was to be a special taille and efforts were to be made to collect debts, arrears of taxes and the spolia of deceased brethren. Fernández de Heredia was appointed to manage these incomes many of which were to be transferred to Avignon by the papal bankers, the Alberti Antichi of Florence ${ }^{2}$. The financial records presented by the Hospital's Receiver-General in the West were normally in Latin ${ }^{3}$. The accounts for $1372 / 3$ produced by Fr. Aimery de la Ribe, who paid a notary to record Treasury business, were in Latin. His financial returns for 1372/3, detailed but very incomplete, were presented to the Master at Beaucaire on 18 October 1374 but not until 27 May 1376, following a careful examination of their content, did the Master and Convent at Rhodes issue a sealed quittance for them ${ }^{4}$.

The accounts for the financial years 1374/5 were in Aragonese, though the opening passage of the parchment onto which they were copied in the form of a quittance to be validated by the Master and Convent was in Latin, as were its closing clauses. The eventual date of the quittance could not be determined when the parchment was ritten out at Avignon, and when it reached Rhodes the leaden bull of the Master and Convent was attached to it but the dating clause was not added. Possibly there was another copy which was sent back to Fernández de Heredia as a receipt while the parchment which survived at Rhodes was kept there for accounting purposes; alternatively, the surviving version was sent back to Avignon but later returned to the

${ }^{2}$ A. LutTrelL, Latin Greece, the Hospitallers and the Crusades: 1291-1440, London 1982, XV, pp. 404-406; see also J. Del AVILLE Le RoulX, Les Hospitaliers à Rhodes jusqu'à la Mort de Philibert de Naillac: 1310-1421, Paris 1913.

${ }^{3}$ J. NISBET, "Treasury Records of the Knights of St. John in Rhodes", Melita Historica, ii no. 2, 1957; Nisbet, pp. 99-101, publishes the accounts of the Hospital's Procurator-General in the West for 15 April 1364 to 26 May 1365, but no other accounts of this type have yet been published.

${ }^{4}$ Item solui notario scriptori negotiorum Thesauri mecum commorani XXV flor' auri, Valletta, National Library of Malta, Archives of the Order of St. John, Cod. 16, no. 53. 
central archives at Rhodes either when Fernández de Heredia became Master in 1377 or on his death in $1396^{5}$.

The annual accounts kept in the West recorded the responsiones, that is the ordinary dues collected by the priors from the commanders for transfer to Rhodes; any arrears which happened to have been paid; the spolia or mortuaris of dead Hospitallers; and occasional incomes from vacant commanderies. In $1373 / 4$ and $1374 / 5$ there was also the special taille fixed at one third of the responsiones. Incomes were channelled through the priors except that, for special historical reasons, a number of Southern Italian commanderies paid their dues directly and not through the priories. Non-payments were often noted, and were so frequent that no invididual annual account represented a complete or nearly-complete record for a financial year. Payments in various currencies were translated into florins current at Avignon. Expenses in the West were for the securing and writing of bulls, for couriers, and for pensions and retaining fees paid to the Hospital's Cardinal Protectors and to a number of Hospitaller and papal officials at the curia. In 1373/4 and 1374/5 there were expenditures on repaying loans advanced, by the Alberti Antichi, on the papal mission of Hospitallers and theologians sent to Constantinople, and on repayments in the West of loans advanced by Latin merchants at Rhodes.

These transactions were set down with a variety of ambiguities. The totals which could be derived from the accounts gave a very incomplete impression of the Hospital's incomes, and there were other revenues collected in Rhodes and Cyprus; furthermore the Master had personal incomes which were separate from those of the Convent of the Order. The payments recorded in the West for the years from 1367 to 1373 averaged about 22,700 florins annually, and those between 1378 and 1399 about 38,500 florins annually ${ }^{6}$. The marginally higher figures for $1373 / 4$ and $1374 / 5$ reflected the extraordinary measures taken for the pas-

${ }^{3}$ Whether the document was registered in the Master's liber bullarum cannot be known as the relevant volume is lost.

${ }^{6}$ Based on details in Nisbet, pp. 102-104. 
sagium against the Turks? The totals were somewhat uncertain, and the accounts often contained mistakes. In 1365 there were complaints that one Receiver-General in the West had omitted 4,000 florins which were missing in cartularijs rationum thesauri $i^{\S}$, while a remark on the obverse of the accounts for $1367 / 9$ again noted an error ${ }^{9}$. Where the sums in the surviving copy of the $1373 / 4$ and $1374 / 75$ accounts did not add up to the totals given, this was apparently because the figures were transferred incorrectly from the original accounts rather than because the totals had been added wrongly in the first place in the accounting kept at Avignon.

Anyone in the Treasury at Rhodes who attempted to analyze these accounts would have found careless copyings and other difficulties. Fluctuations in exchange rates and the costs of exchanging monies must have caused discrepancies. Expenditures on a new register and the mention of a pagina containing figures referred to the records being kept at Avignon ${ }^{10}$. Errors in the parchment sent to Rhodes would have frustrated calculations there, but that particular text was a receipt, not the original accounting record. Nine priores were listed as paying

${ }^{7}$ In 1372/5 a florin de camera was worth 28 sous of Avignon; a florin currens, 24 sous; a florin de sententia, $27 \frac{1}{2}$ sous; 6 florins correntes were worth 5 francs; etc. Except that in 1373/4 the accounts calculated the florin de sententia at $27 \frac{2}{3}$ sous, the equivalencias in the $1373 / 5$ accounts coincided with those in the papal documents, K. SCHÄFER, Die Ausgaben der aposiolischen Kammer unter Johann XXIII. nebst der Jahresbilanzen von 1316-1378, Paderborn 1911, 54*, 59*. Florins correntes were apparently equivalent to, or identical with, those of Provence current in Avignon: H. RoLLAND, Monnaies des Comtes de Provence: XII-XIV siècles, Paris 1956, p. 153-154, 164. Twelve denarii made one sous; one groat or grossus, a silver coin, was apparently worth 2 sous. Sums in the tables are given correct to the nearest florin; brackets indicate calculated or corrected sums; in the notes grossi are converted into sous.

${ }^{8}$ Malta, Cod. 319, f. $3 \mathrm{v}-4$.

9 Malta, Cod. 16, no. $46,48$.

10 An extremely detailed account book for the Western incomes and expenditures from 1378 to 1389 is in Malta, Cods. 48 and 55. In 1372/3 Fr. Aimery de la Ribe, Receiver-General in the West, paid $4-\frac{1}{2}$ florins pro uno libro empto ad copiandum ibidem bullas vel alia negotia Thesauri: Malta, Cod. 16, no. 53 . 
nothing in $1373 / 4$, though some of these paid their arrears in 1374/5; Portugal had paid nothing for nine years. No total was given for $1373 / 4$ but incomes seem to have amounted to 42,230 florins and expenditures to 35,263 , leaving a considerable unaccounted difference which may eventually have reached Rhodes. Of the expenditures, over 5,000 florins went to the Constantinople mission; other expenses in the West amounted to more than 3,000 florins; and over 26,000 florins were paid to the Master, who was then in the West and must have expended part of this sum before reaching Rhodes. The incomes from Italy seem to have arrived in Avignon en bloc, as suggested by equalities in the figures given; they may have been transferred by the Alberti who regularly moved monies for the Hospital". The English Hospitallers, whose returns were listed as nil in the accounts for $1373 / 4$ and $1374 / 5$, may have preferred to send their monies by way of Venice rather than through France'

The returns for 1374/5 were presented less carefully than those for $1373 / 4$. They included special payments imposed on $25 \mathrm{Sep}-$ tember 1374 in order to raise 10,000 florins for the journey to Rhodes of the newly-elected Master, Fr. Robert de Juilly ${ }^{13}$. 46,820 florins were apparently recorded as having been received while sums totalling perhaps 6,611 florins were said still to be owing from Italy, making an overall total of 53,431 florins. Expenditures apparently consisted of 34,278 florins of detailed expenses and a further 17,765 florins paid to the Grand Commander of the Hospital, making a total of 52,043 florins, a figure not unlike that for the incomes. The repayments of monies advanced in Rhodes and sums paid over to the Grand Commander, who presumably transferred them to Rhodes, amounted to 39,908 florins. All these figures were subject to various cautions and reservations, but they did indicate the general order of magnitude of the Hospital's Western incomes.

1 A. LutrRelL, The Hospitallers in Cyprus, Rhodes, Greece and the West, 1291-1440, London 1978, VII 180; VIII 322, 325.

12 LUTTRELL, 1978, V 199; 1982, I 259.

${ }^{13}$ Malta, Cod. 320, f. 32-32v. 


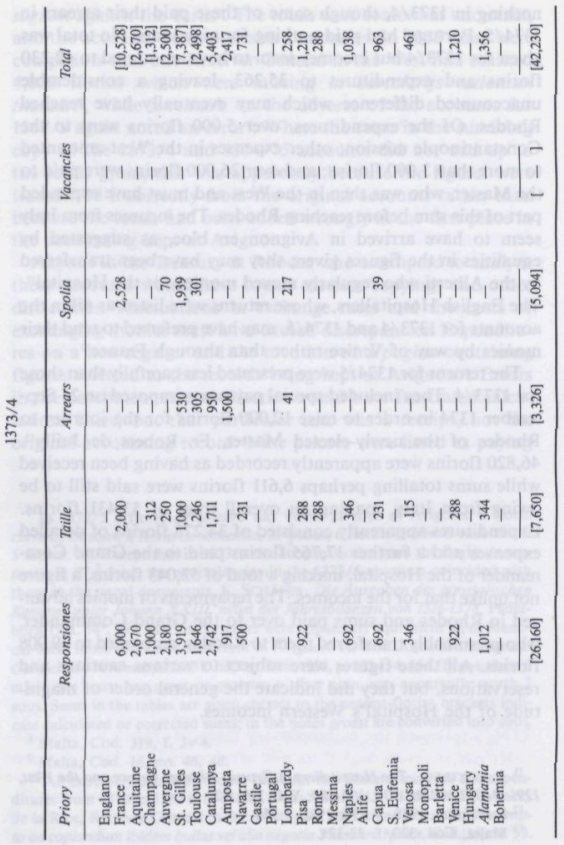




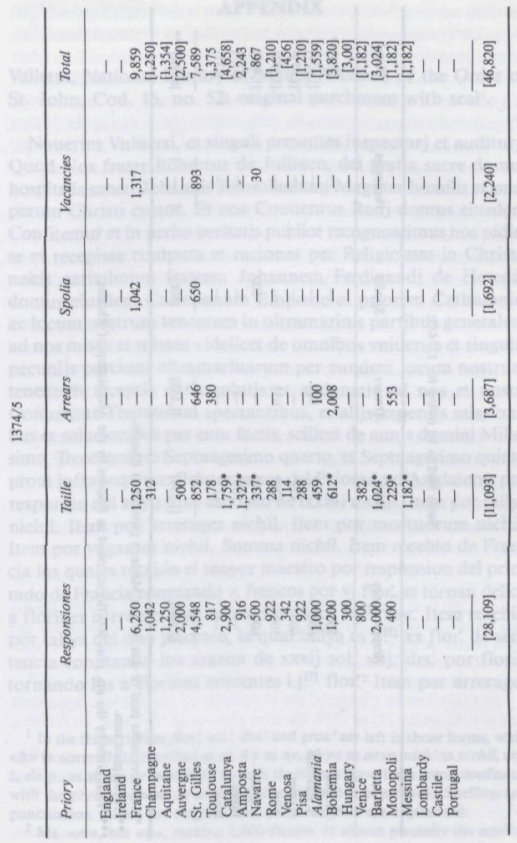




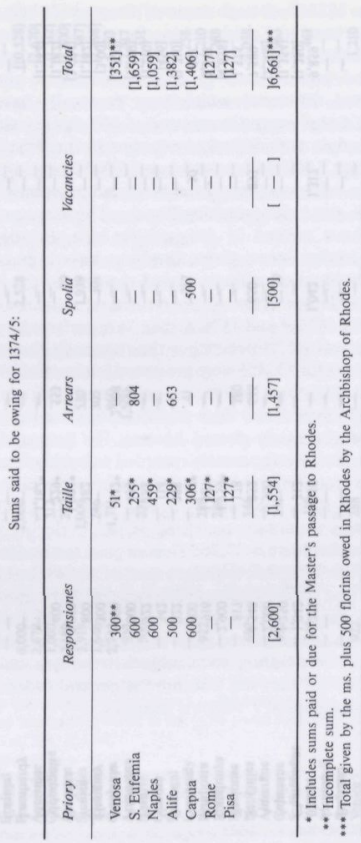




\section{APPENDIX}

Valletta, National Library of Malta, Archives of the Order of St. John, Cod. 16, no. 52: original parchment with seal'.

Nouerint Vniuersi, et singuli presentes inspecturj et auditurj. Quod Nos frater Robertus de Julliaco, dei gratia sacre domus hospitalis sancti Johannis Jerosolimitanj Magister humilis et pauperum Christi custos. Et nos Conuentus Rodj domus eiusdem Confitemur et in uerbo ueritatis publice recognoscimus nos uidisse et recepisse computa et raciones per Religiosum in Christo nobis carissimum fratrem Johannem Ferdinandj de Heredia domus eiusdem Castellanum Emposte et priorem Cathalonie, ac locum nostrum tenentem in ultramarinis partibus generalem ad nos missa et missas videlicet de omnibus vniuersis et singulis pecunijs parcium ultramarinarum per eundem locum nostrum tenentem receptis datis solutis et assignatis ad nos et nostri Conuentus Thesaurum spectantibus, et alijs expensis missionibus et solucionibus per eum factis, scilicet de annjs dominj Millesimo, Trecentesimo Septuagesimo quarto, et Septuagesimo quinto prout infra sequitur. Primerament del Priorado d'Anglaterra por responsio del anyo suso escripto de Ixxiiij nichil. Item por tallya nichil. Item por arrerages nichil. Item por mortuorum nichil. Item por vagantes nichil. Summa nichil. Item recebio de Francia los quales recebio el senyor maestro por responsion del priorado de Francia conptando v. francos por vj flor., et tornan dellos a florines correntes que fazen por todos . $\mathrm{vj}$. $\mathrm{m}$ flor', Item recebio por tallya del dito priorato, la qual tallya es ij $\mathrm{m}$ xx flor' de sentencia conptando los arazon de xxvij sol.' viij. drs.' por florin tornando los a florines correntes i.j! flor.; $^{2}$ Item por arrerages

' In the transcription flor.; sol.; drs.' and gros.' are left in those forms, while «\&" in normally transcribed as $e t, \tilde{n} y$ as ny, an yo as anyo, nich' as nichil, and $\hat{3}$. de $\hat{p}$. as suma de priorado; though there may have been some confusion with the phrase summa de pagina in the original acount book. Spelling and punctuation follow the original, but proper names are capitalized.

${ }^{2}$ Ms. «m», but «ij», making 2,000 florins, is almost precisely the equiva- 
del dito anyo nichil. Item por mortuorum del anyo suso scripto conptando arazon de v. francos por vj flor? et tornandolos a florines corentes vj flor.'

Item por la despuylla de fray G. de Chaucony spitallero del Couent de Rodes ${ }^{3}$, el qual morio en Auinyo en lanyo suso scripto entre dineros et vaxella dargent .m. viiijc xxviij flor.' Item por Vagantes nichil. Summa de priorado .ix! ${ }^{\mathrm{v}} \mathrm{c}$. xxviij flor. ${ }^{4}$ Item recebio del priorado de Aquitania por responsion del dito priorado del anyo suso scrito conptando v. francos por vj florines et fagon flor? correntes $\mathrm{ij} \mathrm{m}^{\mathrm{m}} \mathrm{vj}$ ? lxx flor. Item por tallya nichil. Item por arrerages nichil. Item por mortuorum nichil. Item por vagantes nichil. Item recebio del priorado de Champanya por responsion del dito priorado del anyo suso scripto mill? flor' Item por tallya dek dito priorado del dito anyo es assaber a razon de v. francs per vj flor' fazen de Correntes .iij! xij. flor' vj gros? Summa de priorado .ij. ${ }^{\mathrm{m}} \mathrm{dC}$. Ixx flor.' Arrerages, mortuorum et vagantes nichil. Item recebio, del priorado de Aluernia por la responsio del dito priorado del anyo suso scripto, ij ${ }^{\mathrm{m}} \mathrm{Clxxx}$ flor' Item por tallya del dito priorado del dito anyo .ij 1 . flor' Item per mortuorum del dito anyo Ixx flor' Summa del dito priorado ${ }^{6}, \mathrm{ij}$ ! $\mathrm{V}$. $\mathrm{x}$. flor: ${ }^{7}$ Item recebio del priorado de sant Gilj por responsion del dito priorado del anyo suso scripto .iij: viiij c xviij flor., sol'. xvj. Item por tallya del dito priorado del anyo suso scripto, $\mathrm{M}$ flor' Item por arrerages Vç xxix flor' sol.'

lent of 2,030 florins de sentencia; furthermore on 29 August 1374 the Master gave Fernández de Heredia a quittance for 6,000 florins of responsiones and 2,000 florins of taille from the Priory of France which the Master had receibed directly from the collector of the priory, Malta, Cod. 23,no. 4; Cod. 320, f. $2,11 v, 44 v-45$.

${ }^{3}$ Fr. Guillaume de Chaconin, the Hospitaller of Rhodes, who apparently died in Avignon in 1373 or 1374, was alive there on 22 November 1373: Malta, Cod. 347 , f. 51-51v.

${ }^{4}$ The correct total is 10,528 (not 9,528) florins.

${ }^{5}$ The figures amount to 1,312 florins 12 sous (not 2,670 florins, which was the total for the previous entry for Aquitaine).

${ }^{6} \mathrm{Ms}$. repeats $S$, de $p$.

7 The figures amount to 2,500 (not 2,510 ) florins. 
xvj. Item por mortuorum M. viiij xxxviij flor' sol.' xv. Summa del priorado .vij! ${ }^{\mathrm{iij}}$ xcvj flor' $\mathrm{xj}$ gros.' Item recebio del priorado de Tholosa por responsion del dito priorado del anyo suso scripto .M. vj؟ xlv. flor', xxij sol? Item por talha .jje xlvj flor? .ij. sol' x. Item por arrerages .iijc iiij. flor' xxvj sol' Item por mortuorum iijc flor' xxvij. sol' $x$. drs.' Summa del priorado .ijm iiij! xcvij. flor' $x$. gross. Summa del priorado ixm viij xciij flor? .x. gross." Item recebio del priorado de Catalunya por la responsion del dito priorado del anyo suso scripto. $\mathrm{ij}^{\mathrm{m}}$ vij? slj flor? xiij sol? ij dr?' Item por tallya del dito priorado, $\mathbf{M}$.vj c ij. flor? xviij sol' iij. Item daltra part por tallya del dito priorado, C. viij. flor?' v. sol. xj. Item por arrerages viiij 1 . flor? Summa del prio-

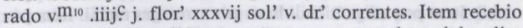
dela Castellania d'Amposta" por responsion dela dita Castellania ${ }^{12}$ del anyo suso scripto es assaber $\mathrm{xj}^{\mathrm{m}}{ }^{\mathrm{m}}$ torneses que fazen de flor' correntes viiij xvj flor' xvj. sol? Item por tallya nichil. ${ }^{13}$ Item por arrerages. m.d. flor' Summa dela Castellania, ij! ${ }^{m}$ iiij! xvi. flor' xvj sol'. correntes. Summa de priorado vijm viij̣ xix. flor' iiij. gros., v. drs'. correntes ${ }^{14}$. Item Recebio por responsion del priorado de Naurra del anyo suso scripto $\mathrm{V}$ c flor? Item por tallya del dito priorado .ijc xxx. flor' xiij sol: iij. dr? Summa del priorado vij? xxx flor' xiij sol.' .iij. dr' Item recebio dei priorado de Castilla por responsion del dito priorado del anyo suso scripto nichil. Item por tallya del dito priorado nichil. Item por arrerages nichil. Item por mortuoris nichil. Item por vagantes nichil. Summa del priorado vij? xxx flor? xiij sol' iij dr.'15 Item recebio del priorado de Portugal por responsion del dito

${ }^{8}$ The figures amount to 7,385 florins 23 sous (not 7,396 florins 22 sous).

${ }_{9}$ This entry of 9,893 florins 20 sous represents the combined totals for Saint Gilles and Toulouse.

${ }^{10}$ Ms: $v$ ?

1 Castellania d'Amposta: the Priory of Aragon.

12 Ms. repeats dela dita Castellania.

${ }^{13}$ Ms. omits nichil.

147,819 florins 8 sous drs.' is the total for Catalunya and Amposta.

15 This entry for Castile, which paid nothing, results from adding Castile and Navarre. 
priorado del anyo suso scripto nichil. Item por tallya nichil. Item por arrerages nichil. Item por mortuoris nichil. Item por Vagantes nichil. Item Recebio por arrerages del priorado de Lombardia xlj flor' ij. sol: iij. Item por la despulla de fay Milan Farina ${ }^{16}$ ij̣ xvij flor' viij sol'. iij. Summa del priorado ${ }^{17}$. ij? lviij flor' $\mathrm{x}$ sol.' vij drs.' Item recebio por responsion del priorado de Pisa del anyo suso scrito. viiijc lxxxviij. flor' sol' iiij. dr' viij. Summa del priorado .m.iĵ̣ x. flor' .x. sol', iiij dr' Item Recebio del priorado de Roma por la tallya del dito priorado del anyo suso scrito .ij! lxxxviij. flor' .iiij. sol.' Summa del priorado m.iiij? xcviiij. flor' correntes .xv. sol', ${ }^{18}$ Item recebio del priorado de Messina por responsion del dito priorado del anyo suso scripto nichil. Item por tallya nichil. Item por arrerages nichil. Item por mortuoris nichil. Item por vagantes nichil. Item Recebio del priorado de Napols por responsion del dito priorado del anyo suso scripto vj: xcj. flor' sol.' xvj. Item por tallya del dito priorado iij xlv. flor' xx sol: Summa del priorado m.xxxvj flor' correntes .xxvj sol. ${ }^{19}$ Item Recebio por responsion del Scambi d'Alif ${ }^{20}$ delanyo suso scripto nichil. Item por tallya nichil. Item por arrerages nichil. Item por mortuoris nichil. Item por vagantes nichil. Item Recebio del priorado de Capua por responsion del dito priorado del anyo suso scripto vjẹ xcj flor' xv. sol.' xj dr?' Item por tallya del dito priorado .ij̣ xxx flor? xiij sol: iij. dr' Item por mortuoris xxxix flor? xiij sol. $x$. dr' Summa del priorado ${ }^{21}$ viiij c Ix flor' xliij sol'. j. dr' correntes. Item Recebio del Priorado de Sancta Eufemia ${ }^{22}$ por responsion del anyo suso scripto nichil. Item por tallya nichil. Item por arrerages nichil. Item por mortuoris nichil. Item por vagantes nichil. Item recebio del priorado de Venosa ${ }^{23}$ por la responsion del dito priorado del anyo

${ }^{16} \mathrm{Fr}$. Milano Farina: unidentified.

${ }^{17}$ Ms. repeats $S$. de $p$.

${ }^{18} 1,498$ florins 15 sous is the total for Rome and Pisa.

19 xxvj sol.' should read $x x x v j$ sol,'

${ }^{20}$ Scambi d'Alif: the Commandery of Alife in the Kingdom of Naples.

${ }_{21}^{21}$ Ms. repeats $S$. de priorado.

${ }^{22}$ Santa Eufemia in Calabria: a commandery, not a priory.

${ }^{23}$ Santa Trinità di Venosa in Apulia: a commandery, not a priory. 
suso scripto .iiic xlv. flor' .xx. sol' Item por tallya .C. xv. flor' vj. sol' .viij. Summa de priorado .iiij lx. flor' xxvij, sol' viij drs. correntes. Primo Recebio del priorado de sant Esteue de Monopoly ${ }^{24}$, por la responsion del dito priorado del anyo suso scripto, nichil. Item por tallya nichil. Item por arrerages nichil. Item por mortuoris nichil. Item por vagantes nichil. Item recebio del Priorado de Barleta por la responsion del anyo suso scripto nichil. Item por tallya nichil. Item por arrerages nichil. Item por mortuoris nichil. Item por vagantes nichil. Item recebio del priorado de Venecia por la responsion del dito priorado del anyo suso scripto .viiij! xxij flor' $V^{\circ}$ ss' iij. dr' Item por tallya del dito priorado .j̣̣ Ixxxviij. flor' sol'. iij. viij. dr.' Summa del priorado ${ }^{25}$. Item recebio del priorado d'Ongria por la responsion del dito priorado del anyo suso scripto nichil. Item por tallya nichil. Item por arrerages nichil. Item por mortuoris nichil. Item por vagantes nichil. Summa de priorado .m.ij! x, flor' x. sol.26 Item Recebio del priorado d'Alamanya por responsion del dito priorado del anyo suso scripto .m. xj. flor' xij sol.' Item por tallya .iij! xliiij flor' vj. sol'. Summa del priorado ${ }^{27}$. Item Recebio por responsion del dito priorado de Boemia del anyo suso scripto nichil. Item por tallya nichil. Item por arrerages nichil. Item por mortuoris nichil. Item por vagantes nichil. Summa de priorado m.iij. lv, flor' xviij sol.' Pagamentos fechos enel anyo de lxxv? Primerament fueron liurados por la companya de los Albertes antigos mercaderos de Florença, a xx. dies de octobre del anyo .m.cc.Ixxiiij. en Auinyo al senyor Maestro del Hospital, es assaber en florines doro de Cambra .xij. ${ }_{\mathrm{ij}}$ ! 1 . Item en florines correntes doro .xij $\mathrm{m}_{\mathrm{ij}} \mathrm{c}$ l. por las manes de Matheu de Vita dela dita companya conptando el flor' de Cambra a xviij sol., e lo

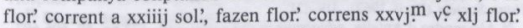
xvj. sol? correntes $^{28}$. Item pago la dita Companya al Cardenal de Mende, al Cardenal de Thoroana, a monss. de Florença, a

\footnotetext{
24 Sant Stefano Monopoli in Puglia: a commandery, not a priory.

25 The missing total is 1,210 florins 10 sous.

26 This sum of 1,210 florins 10 sous is the total for Venice.

27 The total for Alamania is 1,355 florins 18 sous.

28 26,541 florins 16 sous were paid to the Master.
} 
mons. de sant Eustati ${ }^{29}$, es assaber a cascuno dellos por la pension del anyo de Ixxiij. iij̣ flor' doro correns a cascuno que fazen m.ij̣ flor.' correntes. Item pago la dita companya a fray R. Asam procurador en cort de Roma porel el dito senyor Maestro ${ }^{30}$ por la pension del anyo suso scripto, 1. flor' correntes. Item pago la dita companya e los quales fueron pagados enel palacio del papa, es assaber alos porteros dela primera puerta florins viij. Item a los dela i.j. porta flor' xij. Item a los maceres ${ }^{31}$ del papa flor' xxv. Item a los maestres vxeros del papa flor' xxv. Item a los maestres vxeros del papa flor' l. et esto por pension del anyo de Ixxiiij, que fazen en somma, xcv flor' correntes. Item pago la dita Companya a miss' Alexandre Delantella ${ }^{32}$, et a miss. Francisco Bru ${ }^{33}$, et a miss. Jac[omo] Sona ${ }^{34}$ por la pension del anyo de lxxiiij, es assaber a cascuno .I. flor', correntes que fazen, Cl. flor' correntes. Item pago la dita companya a fray Pere Buxon $^{35}$ por la pension suya del a procuracion de la religion de lanyo de Ixxiiij. iij̣̣ flor' correntes. Item pago la dita companya a maestro Feri Casinell procurador del senyor Maestro ${ }^{36}$ por la pension del anyo lxxiij. iiĵ̣ flor. correntes. Item pago la dita com-

29 The Hospital's Cardinal Protectors were Guillaume de Chanac (Mende), Aycelin de Montaigu (Thoroanne), Pietro Corsini (Florençia) and Pierre Flandrin (S. Eusiati).

${ }^{30} \mathrm{Fr}$. Raymond Adizam, later styled presbiter bacallarius in decretis, who was here pensioned as the Master's procurator in the curia; this text suggests that he was possibly the earliest Hospitallers with legal qualifications to hold such a position: cf. Luttrell (1978), XVI 453. His pension was augmented on 20 October 1374: Malta, Cod. 320, f. 50.

31 The servientes armorum seu masserii, the pope's sergeants-at-arms or noble guard: B. Guillemain, La Cour pontificale d'Avignon, 1309-1376: Étude d'une Société (Paris 1962), 419-421.

32 Alessandro d'Antella, advocatus in the curia: ibid, 573 no. 69, 606-607.

33 Francisco Bruni, papal secretary: ibid., 297-299, 568 n. 40, 571, 712.

34 Jacopo da Ceva, papal advocatus fiscal: ibid., 607.

${ }^{35}$ Fr. Pierre Boysson, chaplain and familiarius of the Master, a procurator of the Hospital at the curia in 1370, and by 18 March 1379 Prior of the Convent at Rhodes: Delaville, 151, 164, 212, 214-215, 299; Luttrell (1978), XVI 453.

${ }^{36}$ Ferry Cassinel, doctor in theology and professor of law, a kinsman of the new Master Fr. Robert de Juilly, his procurator in the Priory of France, and in 1374 his procurator at the curia; Delaville, 197; Luttrell (1978), XV1 453. 
panya a fray Aymeric dela Riba $^{37}$ por la pension del anyo Ixxiiij. iiijc flor' correntes. Item pago la dita companya a miss. Ramon Bernat ${ }^{38}$ por la pension del anyo suso scripto .I. flor.' correntes. Item pago la dita companya a Matheu Sobolinj ${ }^{39}$ notario por scripturas xxv flor' correntes ${ }^{40}$. Item fueron pagados a fray Thomas dela orden de los predicadores ${ }^{41}$ por lo viage de Costantinoble .C. florins correntes. Item a el mismo por la dita razon, xlvj flor' correntes xviij sol'. Item pago la dita companya a maestro Bartholomeu Cieras dela orden delos menores $^{42}$ por el viage de Costantinoble es assaber iij de sentencia conptando el flor' a razon de xxvij. sol: viij drs.' que fan flor' correntes .iiij: xxxviij flor', sol.' j. dr.' .iiij. correntes Item pago la dita companya a Maestro Thomas de boço dela orden delos fraires predicadores por el viage de Costantinoble es assaber .iij! lxxx flor' de sentencia conptando el flor' a razon de xxvij sol.' viij. dr' por flor', que fan .iiij! xxviij flor' sol.' .j. dr' iiij correntes. Item pago la dita companya a fray Hesse d'Alamanya ${ }^{43}$, por la messageria de Costantinoble por paga de vj meses es assaber .iiij? lxj. flor' ij. sol., viij correntes. Item pago dotra parte la dita companya al dito fray Hesse por la paga de v. meses por la dita missageria es assaber, vc xviij flor., xviij sol.' correntes. Item pago la dita companya a fray Bertran Flota $^{44}$ por la paga de v. meses por la messageria de Costantinoble es assaber V'c xviij flor? correntes cviij. sol'. Item pago dotra parte al dito fray Bertran Flota por paga de vj meses por la dita

${ }^{37}$ Fr. Aimery de la Ribe, Commander of Raissac, nominated ReceiverGeneral in the West circa 2 February 1372, and previously a procurator of the Hospital in the curia: Delaville, 144 n. 1; Luttrell (1978), XVI 453.

38 Ramon Bernat: unidentified.

${ }^{39}$ Matteo Soboloni, notary: unidentified.

40 Pensions and 25 florins for scripturas amounted to 2,570 florins.

41 Tommaso de Bosolasco, OP: Luttrell (1982), XV 407 n. 68.

42 Bartolomeo Cherrazio, OFM: ibid., XV 407 n. 68.

${ }^{43}$ Fr. Hesso Schlegelholtz. Commander of Freibourg-im-Breisgau: Delaville, 185: Luttrell (1982), XV 407-408.

${ }^{44}$ Fr. Bertrand Flotte, Commander of Naples until December 1374 or a little later, who became Grand Commander of the Convent on 6 May 1375: Delaville, 150 n. 1; Luttrell (82), XV 407-408. 
missageria es assaber .iiij̣ lxj flor' correntes .ij. so.' viij dr' Item pago la dita companya a los de suso nombrados por nolit dela galea en que andaron es assaber V? lxxvj flor.' correntes sol.' ix. drs' iiij. Item pago la dita companya a Johan Corsin ${ }^{45}$ por su prouision dela missatgeria que fizo en Jenoua et en Venecia por los afferes dela missageria de Costantinoble es assaber flor? .vij. Ixvij flor' correntes, .xviiij sol'. Item pago la dita companya a Michel de Rodulfo, et a Luys de Felipo Marin mercaderos de Jenoua, et a miss. Rafael Espindola ${ }^{46}$ por cambio que fezieron alos missageros suso scripto, el qual cambio recebiron a Pera segunt que aparesce per vna scriptura seyellada con .iiij. seyellos de los ditos .iiij missatgeros et pagueren ne en flor' correntes .M.Clij. flor' correntes .xviij sol'? viij dr. ${ }^{47}$ Item posem en paga la dita companya los quales fezieron de messiones en los afferes dela religion es assaber por xviij processos apostolicals las quals fueron embiadas por los priorados dela religion et desto mostraren los jnstrumentes xxxviij flor.' Item por .j. correu, que embiaren en Anglaterra por afferes dela religion, vij flor' .xij sol: Item por correus que embiaron en Francia en Champanya, en Aluernia, en Aquitania por afferes dela religion XV flor.' Item por j. correu que enbiaron en Portugal por los afferes dela religion xxx flor' Item por .j. correu que embiaron en Pulha, et enel Regno ${ }^{48}$ en Jenoua, et en Venecia, en Pisa, et en Florenç .x. flor.' Item por .j. correu que embiaron en Alamanya et en Boemia xxx flor. Item por scripturas et processos en pargamin los quales se fueron delantes delos Cardenals .liiij. flor' Item por bollas, et obliganças que fezieron en la Assembleya que se tuuo en Auinyo. Cxcv. flor' xij sol.' Item por vna bolla de fran-

45 Giovanni Corsini, brother of Cardinal Pietro Corsini, acted for the pope in crusading negotiations at this time: Luttrell (1982), XII 286; XV 400, 407, and A. Benvenuti Papi, "Corsini, Giovannis, Dizionario Biografico degli Italiani, xxix (Rome, 1983), 638-640.

${ }^{46}$ Michele di Rodolfo, Luigi de Filippo Marino, Raffaele Spinola: Genoese.

${ }^{47}$ The Constantinople mission expended 5,479 florins 12 sous plus 728 florins in the $1374 / 5$ accounts, a total of 6,207 florins 12 sous; amend Luttrell (1982), XV 467 n, 68, which gives 6,677 florins. Pera was the Genoese suburb of Constantinople.

48 enel Regno: to the Kingdom of Naples. 
quesa dela Religion la qual bola preso $\mathbf{M}$ [aestr]o Ferri ${ }^{49}$ C.xvj flor' xvj sol., Item por mession que fizo .jo dela companya como ando a Marselha por afferes del Maestro ${ }^{50}$. Item que fueron dados a Duran correu que ando al prior de Nauarra et de Castella por requerir los xxv. flor' Item por .j. correu que aporto letras al valcho ${ }^{51} . x x$. sol:' Item por libros en que escreiuron los comptos dela religion xvj flor?' Item por messiones que fizo Matheu de Vita como ando a Belcaire por aportar moneda al maestro ${ }^{52}$, et por contar con el .vij. flor' los quales messiones totas fazen en summa . $\mathrm{vj}^{\mathrm{c}} \mathbf{j}$. flor' correntes .xij sol.' Item posen en dat [...] los quales pagoron por cambio de los Mil ducados doro los quales fueron pagados alos ambaxadores que fueron en Costantinoble a razon de vij flo'. por cento et son en Summa. Ixx flor' correntes ${ }^{33}$. Reebudas fechas por parte del senyor Castellan d'Amposta prior de Cathalunya, lugartenient del senyor Maestro Couent en todas las partidas daquamar delas monedas delos priorados daquamas segunt que se sieguen. Primerament Reebe del priorado d'Anglaterra por la responsion del dito priorado del anyo suso scripto nichil. Item por tallya nichil. Item por arrerages nichil. Item por mortuoris nichil. Item por Vagantes nichil. Item Recebio del priorado de Yrlanda por responsion del dito priorado del anyo suso scripto nichil. Item por tallya nichil. Item por arrerages nichil. Item por mortuoris nichil. Item por vagantes nichil. Summa de pagina nichil. Item recebio del priorado de Francia por responsion del dito priorado a razon de cinco francos por vj flor.' et fazen de florins correntes .vj?

49 Ferry Cassinel: supra note 36.

50 Amount omitted.

51 valcho?

52 Matteo de Vita of the Alberti Antichi was received by the Master as a confrater of the Hospital at Beaucaire on 22 October 1374: Malta, Cod. 320, f. $49 \mathrm{v}$.

53 Couriers, bulls and 70 florins in exchange expenses apparently amounted to 671 florins 12 sous, making an apparent total of expenses for $1374 / 5$ of 35,262 florins 4 sous. A quittance issued by the Master on 11 October 1374 before he left Avignon showed he had received 35,500 florins for the year: Malta, Cod. 23, no. 4: Cod. 320, f. $44 v-45$ (with inaccurancies). At this point the $1374 / 5$ accounts commence. 
ij. 1 . florins correntes. Item recebio por la tallya del dito priorado de aquellos xij! ${ }^{\mathrm{m}} \mathrm{xl}^{54}$ flor' de sentencia que fazen de sentencia por tallya comptando a razon xxvij sol.' viij drs.' por florin, et ha paga de correntes .M.ij̣ 1 . flor' correntes. Item recebio por vagaciones del dito priorado comptando a razon de v. por vj .M.xl flor' correntes gros.' viij. Item por vagacion dela baylia de Henaut .C.iiij flor' correntes gros.' .ij. Item por vagacion dela baylia de Saint Baubure .C.xiiij flor' correntes gros'. vij. Item por vagacio dela baylia de Orliens xxj flor.' correntes gros'. .iij. Item por la baylia que vago de Mont de sisons xxvj flor' sol.' .j. ${ }^{55}$ Item por mortuoris fray G. de Huyllac ${ }^{56}$.M.xlj flor' correntes viij gros' Summa del priorado de Francia .jx ${ }^{m}$ viij: lviiij flor? iiij gros.' correntes, Item recebio del priorado de Champanya por la responsion del dito priorado del anyo suso scripto .M.xlj flor.' correntes gros' viij. Item por la tallya del dito priorado .iij.. xij flor' et medio correntes. Item arrerages nichil. Item mortuoris nichil. Item vagantes nichil. Item recebio del Priorado de Aquitania por responsion del dito priorado del anyo suso scripto $. \mathrm{m} . \mathrm{ij} \mathrm{c}$ correntes. Item tallya nichil. Item arrerages nichil. Item mortuoris nichil. Item vagantes nichil. Item recebio del Priorado de Aluernia por responsion del dito priorado del anyo suso scripto .ij! correntes, Item por la tallya del dito priorado Vc̣ flor' correntes. Item por arrerages nichil. Item por mortuoris nichil. Item por Vagantes nichil... Item recebio del priorado de Sant Gili por responsion del dito priorado del anyo suso scripto .iiij! ${ }^{\mathrm{m}} \mathrm{v}$. $x l v i i j$ flor' correntes. Item por la tallya viij̣ .lij. flor' gros.' iij. Item por arrerages delos anyos Ixxiij. Ixxiiij. Vc xxij. medio correntes. Item por arrerages delas tallyas delos anyos passados .C.xxiij. gros' viij. Item por vagantes viij c xciij. flor' Item por mortuoris vjẹ l. flor' Summa del priorado de sant Gili que ha pagado vijm

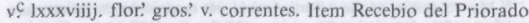

$\left.{ }^{54} \mathrm{Ms}: x i\right]^{m} x l$, perhaps for $x i i{ }^{C} x l$, but the arithmetic gives approximately 1,084 florins. After aquellos is a sign which is repeated in the margin, perhaps to indicate some doubt; the passage is evidently garbled.

55 Exceptionally, the accounts listed the vacancies of four French commanderies: Hainaut, Saint Baubure, Orléans and Mont-du-Soissons.

${ }^{56} \mathrm{Fr}$. Guillaume de Huillac: unidentified. 
de Tholosa por responsion del dito priorado del anyo suso scripto viije xvij. flor' correntes, gros' .ij. $\frac{1}{2}$. Item por la tallya del dito anyo .C.xxix flor' gros' viij. Item pago mas por arrerages deI anyo lxxiiij por las cambras del prior .ij̣ l. flor' gros.' viij correntes. Summa que ha pagado el priorado de Tholosa segunt damunt, M. iij̣̣ Ixxv flor., gros' ij, drs.' xj correntes ${ }^{57}$, Item recebio del dito Priorado ${ }^{58}$ por responsion del anyo suso scripto, ij! viiij̣ flor' correntes, Item por la tallya del dito anyo, m.ij̣ xxiij flor' xj sol: drs.' iij correntes, Item por el passage del senhor Maestre V' xxxv. flor' correntes... Los delas Cambras de Cathalunya M[aestr]e Ferri lo ha preso del anyo Ixxiiij.lxxv. Summa, que ha pagado Cathalunya .iiij! ${ }^{m}$ 'c lxviij flor? xj sol.' drs' iij ${ }^{59}$. Item recebio dela Castellania d'Amposta por la responsion del anyo suso scripto viiij! xvj flor., gros? iiij. correntes. Item ha pagado de tallya .VC ' lxxvj flor' gros. iij. drs.' xvj correntes. Item por el passage del senyor Maestre .ije l. flor' correntes, Item por las cambras del senyor Maestre .Vc flor. Summa que ha pagado lo Castellan d'Amposta entre todo $\mathrm{ij}^{\mathrm{m}} \mathrm{ij}^{\mathrm{c}}$. xlij.flor'. correntes gros'. viij drs'. .xvj. Item recebio del priorado de Nauarra por responsion del dito priorado del anyo suso scripto .$v$ ' flor' correntes Item por la tallya .ij! xxv flor' gros'. j. drs' xvj. Item por el passage del senyor Maestre .C.ij. flor' correntes. Item por vagantes .xx. flor' correntes. Summa que ha pagado el priorado de Nauarra segunt que desuso viij: Ixvij flor: correntes gros.' j. drs. xvj. Item fazo Reebuda los quales recebiron la companya delos Albertes del priorado de Roma por la responsion del dito priorado del anyo de lxxv? viiij c xxij. flor?' correntes sol' v. dr' .iij. Item que Reebiron la dita companya por tallya del dito priorado de Roma .ijc Ixxxviij flor' correntes sol' .iiij dr' viij. Item faze reebuda los quales reebiron los ditos Albertes del priorado de Venosa por la reponsion del dito priorado del anyo de Ixxv .iij? xlij flor' correntes, gros' iiij $\frac{1}{2}$. Item por

57 The figures given amount to 1,296 florins 4 sous (not 1,375 florins 4 sous 11 drs?').

58 ie. Priory of Catalunya.

59 The figures given amount to 4,658 florins 11 sous 4 drs? (not 4,668 florins 11 sous 4 drs'). 
la tallya del dito priorado .C. xiiij flor' correntes gros? j $\frac{1}{2}$. Item faze reebuda los quales recebiron la dita companya delos Albertes por la responsion del priorado Pisa del anyo lxxv. Viiij . xxij flor.' correntes, sol.' v. dr.' iiij. Item por la tallya del dito priorado .ij: Ixxxviij flor' correntes gros.' ij. dr' .viij... Las quantitas suso scriptas son stadas assignadas porell Castellan d'Amposta al grant Comandador ${ }^{60}$. Primo por la responsion de Alamanya .M. flor' Item por la tallya iijc vj. flor' Item por arrerages .C. flor'. Item por el passage del senyor Maestro .Cliij flor' Item por la responsion del priorado de Bohemia del anyo de Ixxiiij. M.ijc flor' Item dela tallya .iiij̣ viij flor? Item la responsion del anyo de lxxv. M.ij! flor' Item dela tallya .iij ç viij flor' Item de arrerages .iiij̣ flor' Item del passage del senyor M[aestr]e .ij̣ iiij. flor.' Item la responsion del priorado de Vngria los quales han recebidos el prior de Venecia .iijc flor' Item la responsion del priorado de Venesia viij c flor' Item por la tallya .ij̣ lv. flor.' Item del passage del senyor M[aestr]e .C.xxvij flor. $\frac{1}{2}$. Item del priorado de Barleta por la responsion que non de ha pagado ren de todo el tiempo que ha tuuido lo priorado, et el grant comendador ha firmado porell .ij! ${ }^{\mathrm{m}}$ flor.61 ${ }^{9}$ Item por la tallya del anyo de Ixxiiij.lxxv. viij xvj flor? Item porel passage del senyor Maestro ij̣ viij. flor' Summa de pagina delas assignaciones fechas viiij: ${ }^{m}$ viii l Ixxxv flor'. $\frac{1}{2}^{62}$. Sant Esteue de Monopoli Ja responsion del anyo de Ixxiiij .iiij̣ flor' Item por la tallya .C.liij. flor?' Item por la responsion del anyo de lxxv .iiije flor', Item por la tallya .C.liij flor.' Item porel passage del senyor M[aestr]e lxxvj

60 The Grand Commander of the Convent was Fr. Bertrand Flotte nominated on 6 May 1375: Delaville, 150 n. 1; supra note 44.

${ }^{61}$ The Prior of Barletta at least between 25 November 1373 and about 30 October 1375 was Fr. Raymond de Sabran: Lettres Secrètes et Curiales du pape Grégoire XI (1370-1378) relatives à la France, ed, L. Mirot - H. Jassemin, 5 fasc. (Paris 1935-1957), nos. 3137, 3779. The Grand Commander apparently pledged to pay the 2,000 florins.

62 The pagina contained the monies assigned to the Grand Commander, that is $9,885 \frac{1}{2}$ florins (to be corrected to $9,732 \frac{1}{2}$ florins) for Alamania, Bohemia, Hungary, Venice and Barletta, assigned to the Grand Commander who was probably charged to collect them in Italy, the monies from Alamania and Bohemia often being sent to Venice. 
flor: $\frac{1}{2}$. Venosa ha pagado responsion et tallya a los Albertes, et deue porel passage de mons. lo m[aestr]e .lj. flor' Item deue dela responsion ,iij. flor.' Item deue .iij. flor' ${ }^{63}$. Santa Eufemia deue de ij. anyos responsion et tallya, et faze por .ij. anyos la responsion . $\mathrm{vj}$ C flor' Item por tallya .ij $\mathrm{C}$ iij flor.' ${ }^{64}$ Item porel passage del senyor M[aestr]e .lj. flor' Napol de Responsion vj? flor' Item de tallya .iij. $\mathrm{c}$ vj. flor. Item porel passage del senyor M[aestre] .cliij flor' Los Escambis d'Alif deue dela responsi del anyo de Ixxiiij.$v^{c}$ flor' Item por tallya .Cliiij. flor' Item porel passage del senyor Maestro .Ixxvj flor.' Item por la responsion del anyo de Ixxv? Vc flor' Item por la tallya .Cliij flor'... Capua de responsion.$v j$ c flor' Item por tallya .ij c iiij. flor' Item porel passage del senyor Maestre .C.ij. flor? Roma ha pagado responsion, et tallya deue por el passage del senyor Maestre .C.xxvij flor' $\frac{1}{2}$. Pisa ha pagado responsion tallya alos Albertes, et deue solament porel passage del senyor M[aestre] .C.xxvij flor' $\frac{1}{2}$. Mescina no ha pagado res delos anyos de lxxiiij et Ixxv, et por la tallia, et por lo passage del maestre M.C.Ixxxij. flor.' $\frac{1}{2}$. Lombardia no ha pagado res delos anyos de Ixxiiij, Ixxv mas esta hombre en sperança de auer ne alguna cosa. fray Richardo Caracho de Chichano tiene por mortuor[is] V' flor.65 Item que son stados inprestados a larceuispo de Rodas lo quales deue pagar en Rodas . $V$ ? flor' Castiella non ha pagado res despues que esti prior $^{56}$ tiene el priorado. Portugal deue de ix anyos que non ha pagado res... Aquestos son los pagamientos et assignaciones fechas porel senyor Castellan delas monedas de suso scriptas del anyo de lxxv. Primerament ha pagado dela moneda de Francia ala cambra de nuestro senyor el papa porel deudo que mons.

${ }^{63}$ Venosa had paid ist dues (supra) and can scarcely have owed two sets of only three florins; the copyist must have erred, and 300 florins may have been the sum owed.

64 This perhaps meant that Santa Eufemia owed 600 plus 204 florins each year for two years.

${ }^{65} \mathrm{Fr}$. Riccardo Caracciolo, Commander of Cicciano in the Priory of Capua: Delaville, 249.

${ }^{66} \mathrm{Fr}$. Lop Sánchez de Somoza, destituted in 1375 for his refusal to pay: ibid., 195-196. 
el Maestre deuie ala cambra del papa .V!' flor' de Cambra que ualen conptando v. francos por vj flor', et ualen flor' correntes V'm viij! xxxiij flor' Esti pagamento fue fecho porque la cambra fizo arestar las monedas de Francia entroque se pagaron delos ditos V!m flor' de Cambra. Item ha pagado ala companya delos Albertes los quales fueron deuidos a ellos porel prestamo que se fizo delos xiiij! ${ }^{\mathrm{m}} \mathrm{v}$ ? flor' que priso mons. el Maestro por al Couent, et por otras expensas fachas porels ditos Albertes. iij. ${ }^{m}$ Ixiiij flor: ${ }^{67}$ Item pago por mandamento de mons lo M[aestre], et del Couent segunt paresce por bulla de mons, el maestro, et del Couent, la qual fue dada en Rodas los cuales fueron pagados a algunos mercaderos segunt aparesce por carta fecha por m[aestre] Antonj not[ario] ${ }^{68}$, florines de Cambra, iij ${ }^{m}$ que

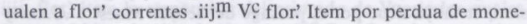
das .iiij flor.' Item a pagado per mandamiento de mons. lo m[aestre] et del Couent segunt paresce por bulla de mons. el maestro et Couent la qual fue dada en Rodas los quales fueron pagados alos mercaderos segont paresce por carga publica fecha por not[ario] flor' de Cambra .vj ${ }^{\mathrm{m}}$ vij $^{\mathrm{c}}$ Ixxxv. que fazen flor' correntes vij! $\mathrm{ix}$ ' xv flor' gros' .x. Item pago alos freres menores et predicadores que fueron en Costantinoble por mandamiento de nuestro senyor el papa, ultra la somma que les fue pagada en lanyo de Ixxiiij vij. $x x$ viij flor. Item pago a mons. Anchelet ${ }^{69}$ segunt paresce por la bulla de mons. el M[aestre] .V c flor.' Item pago por la pension de iiij Cardenals m.ij̣ flor' Item pago alos officiales de nuestre senyor el papa xcv. flor'. Item pago a iij aduocados, et a miss. Frances Bru ${ }^{70}$, a cascu .l. flor', fazen ij! flor? Item pago a M[aestr]e Matheo procurador delas contradichas ${ }^{\text {?] }}$ xxv. flor' Item pago al procurador general de Cort de Roma,

${ }^{67}$ These 24,500 florins appeared above as 26,541 florins 26 sous, and the 3,064 florins of charges listed here presumably included interest on money loaned; al Couent should probably read el Couent.

68 Antonio notario: unidentified.

${ }^{69}$ Mons. Anchelet, possibly the papal scribe Ancelin Martin: Guillemain, $342,366$.

${ }^{70}$ Francesco Bruni, papal secretary.

${ }^{71}$ Matheo, advocatus in the audientia litterarum contradictarum. 
iij flor.? ${ }^{72}$ Item pago por bollas processos correus andantes por diuersas partidas por los negocios dela religion clvj flor' gros' .j. Item pago a Loys Tallaborch ${ }^{73}$ por la pension a el assignada por mons. lo maestre .xx. flor: ${ }^{74}$ Item que ha pagado manualment al grant comendador segunt aparesce por carta f[ech]a conptando v. francos, por vj flor' et fazen flor' correntes $v j$ m vje xxix flor.' Item pago al grant Comendador dotra parte por man de fray Aymeric dela Riba .m. v⿳̣ xcvij flor' gros' vj, drs' .iiij. Item pago al dito grant Comendador dotra part .ij! ${ }^{\mathrm{C}}$ ? flor. ${ }^{75}$ Summa que son las pagas fechas del anyo de lxxy a florines xxxiiij! ${ }^{\mathrm{m}}$ ij xxviij gros. ${ }^{76}$ Item las assignaciones fechas al grant comandador las quals son de suso scriptas .xvij! vij $^{\mathrm{c}} \mathrm{lxv}$. flor' "7. Que quidem computa et rationes, ut premittitur si cuisa et recepta ipsa tenore presencium ratificamus approbamus et omologamus et dictum fratrem Johannem Ferdinandj de Heredia nostrum locumtenentem, ac omnia bona sua et arnesia presentia et futura quitamus liberamus et absoluimus per presentes.

\section{[a leaden bull attached with string is inscribed:] BVLLA MAGISTRI ET CONVENTVS / HOSPITALIS IHERVSALEM}

72 One of the Hospital's procurators, magister Matteo de Lucha, was confirmed as procurator in the curia on 10 October 1374: Malta, Cod. 320, f. 45. The total for pensions was 1,795 florins.

${ }^{73}$ Lodovicus Tallebrusus, inhabitant of Avignon, was granted a life pension of 30 florins a year by the Master on 22 October 1374: Malta, Cod. 320, f. 50 . He was campsor or moneychanger: Cod. 16, no. 53 .

${ }^{74}$ Couriers, bulls and Tallaborch's pension amounted to 186 florins 2 sous.

${ }^{75}$ Payments to the Grand Commander amounted to 9,721 florins 12 sous $1 d r^{\prime}$

${ }^{76}$ These expenses included 4 florins lost, 11,415 florins 20 sous in repayments of monies advanced at Rhodes, and 728 florins to the friars who went on the Constantinople mission. The total of 34,277 florins 22 sous (not 34,228 florins ? sous) seems approximately correct.

77 These 17,765 florins do not seem to be cwritten above" (suso scriptas) and remain somewhat inexplicable; they probably represented the balance between incomes and expenses, since 34,277 plus 17,765 amounted to 52,043 florins while incomes apparently amounted to 53,451 florins, if monies said to be owing were in fact added to those paid. 